\title{
BMJ Open Implementation of the guidelines for targeted temperature management after cardiac arrest: a longitudinal qualitative study of barriers and facilitators perceived by hospital resuscitation champions
}

\author{
Young-Min Kim, ${ }^{1}$ Seung Joon Lee, ${ }^{2}$ Sun Jin Jo, ${ }^{3}$ Kyu Nam Park ${ }^{1}$
}

To cite: Kim Y-M, Lee SJ, Jo SJ, et al. Implementation of the guidelines for targeted temperature management after cardiac arrest: a longitudinal qualitative study of barriers and facilitators perceived by hospital resuscitation champions. BMJ Open 2016;6:e009261. doi:10.1136/bmjopen-2015009261

- Prepublication history and additional material is available. To view please visit the journal (http://dx.doi.org/ 10.1136/bmjopen-2015009261).

Received 30 June 2015 Revised 10 November 2015 Accepted 8 December 2015

CrossMark

\footnotetext{
${ }^{1}$ Department of Emergency Medicine, College of Medicine, The Catholic University of Korea, Seoul, Korea

${ }^{2}$ Department of Emergency Medicine, Myongji Hospital, Goyang-si, Gyeonggi-do, Korea ${ }^{3}$ Department of Preventive Medicine, College of Medicine, The Catholic University of Korea, Seoul, Korea
}

Correspondence to Dr Young-Min Kim; emart@catholic.ac.kr

\section{ABSTRACT}

Objectives: To identify the barriers to and facilitators of implementing guidelines for targeted temperature management (TTM) after cardiac arrest perceived by hospital resuscitation champions and to investigate the changes in their perceptions over the early implementation period.

Design: A longitudinal qualitative study (up to 2 serial semistructured interviews over 1 year and focus groups). The individual interviews and focus groups were transcribed and coded by 2 independent assessors. Contents were analysed thematically; group interaction was also examined.

Setting: 21 hospitals, including community and tertiary care centres in South Korea.

Participants: 21 hospital champions (14 acting champions and 7 managerial champions).

Results: The final data set included 40 interviews and 2 focus groups. The identified barriers and facilitators could be classified into 3 major themes: (1) healthcare professionals' perceptions of the guidelines and protocols, (2) interdisciplinary and interprofessional collaboration and (3) organisational resources. Lack of resources was the most commonly agreed on barrier for the acting champions, whereas lack of interdisciplinary collaboration was the most common barrier for the managerial champions. Educational activities and sharing successfully treated cases were the most frequently identified facilitators. Most of the participants identified and agreed that cooling equipment was an important barrier as well as a facilitator of successful TTM implementation. Perception of the guidelines and protocols has improved with the accumulation of clinical experience over the study period.

Conclusions: Healthcare professionals' internal barriers to TTM implementation may be influenced by new guidelines and can be changed with the accumulation of successful clinical experiences during the early implementation period. Promoting interprofessional and interdisciplinary collaboration through educational activities and the use of cooling

\section{Strengths and limitations of this study}

- The strengths of this study include the investigation of perceived barriers and of perceived facilitators to implementing the guidelines for targeted temperature management after cardiac arrest.

- This study investigated longitudinal changes in the perceived barriers and facilitators over the early implementation period.

- This study identified the differences in the perceived facilitators and barriers between the champions who have different roles and responsibilities in the respective hospitals.

- One limitation of this study was that only emergency physicians participated in this study because most of the hospital resuscitation champions for targeted temperature management in South Korea are emergency physicians.

- Although most of the barriers identified in this study were similar to those of previous studies, clinicians in different countries or under different systems might have different perceptions and opinions.

equipment with an automated feedback function can improve adherence to guidelines in hospitals with limited human resources in critical care.

\section{INTRODUCTION}

Targeted temperature management (TTM) can improve the chance of survival with good neurological outcomes of patients resuscitated from out-of-hospital cardiac arrest (OHCA) and was strongly recommended in the 2010 guidelines. $^{1}{ }^{2}$ Despite this strong recommendation, the implementation rate of TTM varies by country. ${ }^{3-9}$ Although the 
number of hospitals adapting TTM has increased annually since the 2010 guidelines were provided, the overall implementation rate is still low in many countries. ${ }^{6-9}$

Understanding the barriers to and facilitators of implementing the guidelines is important for developing effective implementation strategies. Despite the evidence-based resuscitation guidelines that have been updated every 5 years, an important gap remains in our understanding of effective strategies for implementing the guidelines. ${ }^{10}$ Although many previous survey studies have attempted to investigate TTM use and barriers to its implementation, there are few qualitative studies available that further elucidate why clinicians do not adhere to TTM guidelines and what influences their behaviour and practice. Since Brooks and Morrison ${ }^{11}$ suggested specific barriers and barrier-specific interventions for implementing TTM after OHCA, only one qualitative study using semistructured interviews has been reported. ${ }^{12}$ Therefore, further research is necessary to better understand the barriers to and facilitators of TTM implementation and the changes in clinicians' perceptions over the implementation period.

The aim of this study was to identify the barriers to and facilitators of implementing guidelines for TTM after cardiac arrest that are perceived by hospital resuscitation champions and to investigate the changes in the perceived barriers and facilitators over 1-year implementation period.

\section{METHODS}

\section{Study design, setting and participants}

We conducted a longitudinal qualitative study using serial semistructured individual interviews and focus groups with hospital resuscitation champions who worked in the emergency department (ED) or intensive care unit (ICU) in various sized hospitals across South Korea. Local resuscitation champions who participated in a TTM training course were recruited for the study. The training course was a full-day, simulation-based training programme for hospital TTM champions. The study period was from December 2010 to February 2012.

Purposive sampling was used to select participants who were the most likely to work in TTM implementation at each hospital. Each participant voluntarily participated in the study after receiving information about the study and confidentiality measures (including anonymity and data security) and providing informed consent.

\section{Data collection}

All individual interviews were conducted via telephone by an investigator (Y-MK) who was the director of the TTM training course and had experience in qualitative research with an interview guide (see online data Supplement 1-A). The interview guide was developed with the help of an experienced qualitative researcher (SJJ). The guide was based on a framework for improving physician adherence to clinical practice guidelines proposed by Cabana et $a l^{13}$ and on strategies for TTM implementation proposed by Brooks and Morrison. ${ }^{11}$ The guide was tested with local champions who did not participate in the study, and the guide was revised before starting the first interview.

The first interview session was conducted over a 2-month period from 6 months after the TTM training course. Each interview was recorded electronically and transcribed by an independent assistant. The participant verified the accuracy of the transcription and clarified any inaudible passages. Any unrecorded interview was summarised with field notes written by the interviewer during the interview and verified by the participant. During the first interview session, the sample size for the study was determined using the concept of thematic saturation, a standard method for sample size determination in a grounded theory-based qualitative study. ${ }^{14}$ To identify the changes in the perceived barriers and facilitators during the early implementation period, we repeated the interviews with a partially modified interview guide (see online data Supplement 1-B) at 12 months after the TTM training course with the same analysis protocol.

Two focus group discussions were conducted separately with purposively sampled participants divided according to their roles (acting or managerial champions) during the implementation process at 12 months after the TTM training course. Two types of champions were classified according to the definition suggested in a previous report. ${ }^{15}$ 'Acting champions' were front-line clinicians, and 'managerial champions' were responsible for managing clinical departments and wards or units. Each focus group session was guided by a moderator (Y-MK) based on a pre-established interview guide (see online data Supplement 2) in a meeting room. An independent note-taker participated in the discussions and observed, took field notes and recorded the interview. The note-taker transcribed the recordings and field notes of the two focus group sessions according to a transcription guide. All participants verified the accuracy of the transcripts and clarified any non-verbal expressions and their responses during the discussion.

Illustrative quotations were chosen from the interviews or focus groups, as is standard practice in qualitative research. ${ }^{16}$ To ensure confidentiality, all quotes from participants were de-identified. Quotations marked with '(A)' indicate a participant who had the role of acting champion; '(M)', a participant who had the role of managerial champion.

\section{Data analysis}

The transcripts were analysed using the qualitative content analysis method of Graneheim and Lundman ${ }^{17}$ with the six phases of thematic analysis of Braun and Clarke. ${ }^{18}$ First, the transcripts were read repeatedly to grasp their overall meaning. Then, meaningful words, sentences and phrases were extracted from the interviews or focus groups to produce meaning units. The 
produced meaning units were subsequently summarised into a conventional form termed the condensed meaning unit. The condensed meaning units were then grouped together on the basis of their similarity and categorised into subthemes. These subthemes were further condensed and consolidated to elicit themes. Furthermore, the list of analytic questions proposed by Stevens ${ }^{19}$ for analysing interactions within a focus group was used to carefully investigate the interactions among participants during the focus groups. The above methods were used to grasp themes on which the study participants both agreed and disagreed. The results of the analysis were subsequently reflected in the final theme elicitation process.

To increase the validity of the qualitative study results, various methods, in accordance with the general principles of a qualitative study, were used during the data collection and analysis. ${ }^{16}{ }^{20}$ First, to enhance the reliability of the data, resuscitation champions from hospitals of various sizes were included. Then, the triangulation method of analysis was applied by including acting and managerial champions as participants. During the interviews and focus groups, a facilitator trained according to a prepared guide asked open-ended questions to elicit free responses regarding the participants' experiences and opinions on various situations and topics. To preserve data accuracy, all interview transcripts were transcribed by a third research assistant from the recordings of the interview and field notes. The resultant transcripts were reviewed by the participants to establish respondent validation. Two researchers (Y-MK, SJL) independently coded the transcripts and elicited themes according to qualitative content analysis guidelines to enhance the reliability of the data analysis and interpretation. The resultant extracted meaning units, subthemes and elicited final themes were reviewed again by a co-investigator and a qualitative study expert (SJJ); triangulation was also used to modify and supplement the data analysis and further enhance the reliability of the results.

\section{RESULTS}

\section{Participants}

In total, 34 hospital resuscitation champions (26 physicians and 8 nurses) participated in the TTM training course. Among them, 26 physicians who were the most likely to work in the TTM implementation process at each hospital and agreed to participate in the study were randomly contacted. Thematic saturation in the first interview session occurred after 21 interviews. The characteristics of the 21 participants recruited for the study and their institutions are listed in table 1 . Table 2 presents the patient volume and TTM applied at the participating hospitals during the study period. All individual interviews and focus groups were included in the final analysis. Two interviews were not recorded and transcribed, one due to participant preference and one
Table 1 Characteristics of the participants and their institutions

\begin{tabular}{llr}
\hline Classification & Category & n (\%) \\
\hline Sex, male & & $19(90)$ \\
Age, years & $30-39$ & $15(71.4)$ \\
& $40-49$ & $6(28.6)$ \\
Specialty & Emergency medicine & $21(100)$ \\
Position & Managing physician & $7(33.3)$ \\
& Acting physician & $14(66.7)$ \\
Clinical experience, & $5-9$ & $10(47.6)$ \\
years & $10-14$ & $8(38.1)$ \\
& $>15$ & $3(14.3)$ \\
Type of hospital & Academic/tertiary & $9(42.9)$ \\
& Academic/secondary & $11(52.4)$ \\
Number of hospital & Community/secondary & $1(0.5)$ \\
beds & $400-599$ & $4(19.0)$ \\
& $600-799$ & $7(33.3)$ \\
Number of patients & $800-1000$ & $10(47.6)$ \\
per an ED nurse & 5 & $4(19.0)$ \\
& 7 & $3(14.3)$ \\
Full-time intensivist, & 8 & $10(47.6)$ \\
yes & & $4(19.0)$ \\
Number of patients & 1 & $3(14.3)$ \\
per ICU nurse & 2 & $2(9.5)$ \\
& 3 & $2(9.5)$ \\
\hline ED, emergency department; ICU, intensive care unit. & $7(33.3)$ \\
& &
\end{tabular}

due to technical problems with the recording equipment. Fourteen participants, including seven managerial champions and seven acting champions, participated in two focus group discussions (see online data Supplement 3, figure 1).

\section{Implementation efforts and TTM implementation in the} participating hospitals over the 1-year period

In total, three longitudinal surveys were performed during the study period (see online data Supplement 3, figure 1). Implementation efforts such as conferences, educational activities, protocol development and team building in the participating hospitals were progressively increased over the 1-year implementation period (see online data Supplement 3, figure 2). At the baseline, only 5 hospitals $(23.8 \%)$ had their own TTM protocols, but 18 hospitals $(85.7 \%)$ had protocols during the implementation period. Notably, only four hospitals (19\%) built postcardiac arrest care teams during the implementation period.

The number of TTM cases in $16(84 \%)$ of the 19 hospitals that responded to all surveys increased during the implementation period compared with that in the previous year. The TTM implementation rates and the proportion of the TTM applied cases among the OHCA patients with return of spontaneous circulation (ROSC) also increased in the 16 hospitals (84\%) during the 
Table 2 Patient volume and TTM applied at the participating hospitals during the study period

\begin{tabular}{llr}
\hline Classification & Category & $\mathbf{n}(\%)$ \\
\hline Number of patients & $<100$ & $5(23.8)$ \\
with OHCA & $100-199$ & $9(42.9)$ \\
& $200-299$ & $6(28.6)$ \\
Number of patients & $>300$ & $1(0.5)$ \\
with ROSC & $20-39$ & $4(19.0)$ \\
& $40-59$ & $4(19.0)$ \\
Number of patients & $>60$ & $8(38.1)$ \\
treated with TTM & $5-9$ & $5(23.8)$ \\
& $10-14$ & $8(38.1)$ \\
TTM indicated initial & $>15$ & $5(23.8)$ \\
rhythm & Shockable rhythm only & $5(23.8)$ \\
TTM duration, $\mathrm{h}$ & All rhythm & $3(14.3)$ \\
& 24 & $17(81.0)$ \\
TTM target & $32-34$ & $2(9.5)$ \\
temperature, ${ }^{\circ} \mathrm{C}$ & & $19(90.0)$ \\
Main TTM device & Surface cooling without & $19(90.0)$ \\
& an automated feedback & \\
& function & \\
\hline OHCA, out-of-hospital cardiac arrest; RosC, return of & \\
spontaneous circulation; TTM, targeted temperature management.
\end{tabular}

implementation period (see online data Supplement 3, figure 3).

\section{Perceived barriers to and facilitators of implementing TTM after cardiac arrest}

The final data set included 40 interviews and 2 focus groups. Perceived barriers to and facilitators of TTM implementation were categorised into three major themes. These themes were as follows: (1) healthcare professionals' perceptions of the guidelines and protocols, (2) interdisciplinary and interprofessional collaboration and (3) organisational resources and process. Table 3 summarises these themes, subthemes and relevant quotes.

The first major theme was healthcare professionals' perceptions of the TTM guidelines and protocol. Many healthcare professionals, especially doctors in specialties other than emergency medicine, were unaware of the guideline changes. Even if they knew the guideline changes, the participants also perceived that either the lack of a protocol or unfamiliarity with the existing protocol was another barrier to them. In addition, a lack of education contributed to the difficulties in TTM implementation. Consequently, the participants perceived that various forms of education inside and outside the hospital would help overcome the barriers.
The initial clinical experiences of the healthcare professionals also affected their perception and implementation efforts. In the case of experiencing negative adverse events during the early implementation period, the participants often tended to have negative perceptions of TTM. In contrast, many participants emphasised that the publicity of successful cases altered their colleagues' perceptions of TTM and positively influenced the adoption of TTM.

The second major theme was interdisciplinary and interprofessional collaboration. The lack of collaboration among the department chairs and faculty of related departments was perceived as an important barrier. In particular, champions in managerial positions at large-sized, old hospitals identified this factor as the most important barrier (table 4). In contrast, champions in relatively small-sized, newer hospitals did not consider this factor to be a barrier because interdisciplinary collaboration was relatively sound in their hospitals. Furthermore, multidisciplinary and interprofessional conferences to discuss successfully recovered cases and share the experiences were identified as a facilitator.

The third major theme, organisational resources and processes, was the most frequently mentioned and agreed-on major theme. Since postcardiac arrest care is a resource-intensive practice, a lack of manpower and an increase in workload were believed to be a major barrier. In particular, acting champions who are the primary providers of bedside treatment emphasised this theme as an important barrier (table 4). The participants agreed that the absence of cooling equipment with an automated feedback function was an important barrier. In the same context, many participants also perceived the use of cooling equipment with an automated feedback function as a facilitator.

Another important issue was low medical treatment fees and high expenses for disposable supplies. Since a specific treatment fee for TTM after cardiac arrest has not been established in South Korea, the participants worried that the cost of expensive equipment could not be optimally recouped. Furthermore, in some small cities, even when hospitals were equipped with the cooling equipment, the high costs of disposable supplies prevented TTM use.

Championship and leadership were also perceived as an important barrier and facilitator. Some participants mentioned that the dedicated and passionate efforts of a champion facilitated the TTM implementation. Some participants regretted that they did not actively work as champions due to various barriers in their hospitals. Furthermore, a positive attitude and the support of hospital leadership and department chairs were perceived to facilitate TTM implementation.

\section{Changes in the perceived barriers and facilitators over the 1-year implementation period}

During the study period, participants with successful experiences adhered more strongly to their TTM 
Table 3 Identified barriers to and facilitators of the implementation of the therapeutic hypothermia guidelines and illustrative quotes

Themes and subthemes

Barriers

Facilitators

Quotes

Healthcare professionals' perception of the guidelines and protocols of TTM

Knowledge and Lack of awareness attitudes

Disagreement with

Educational activities (internal guidelines Negative attitudes and external) towards the guidelines Publicity for "The staffs of the respective clinical departments are only Lack of familiarity with protocol Lack of publicity successfully treated cases Changes in recommendation irritated, ignorant, and uninterested in post-cardiac-arrest patients" (A)

"I think that there was a big change. Although the other specialists do not know the exact details of the guideline changes, they now at least agree that they should implement...well...something should be done for these patients" (A)

"It went much better, because a consensus was formed after conducting an Emergency Grand Round within our hospital. Of course, outside educational symposia and conferences also helped..." (A)

Clinical experiences

Lack of clinical experience Influence of prior experiences of adverse events
Experience with successfully treated cases

"Until now, we only experienced post-cardiac-arrest patients with poor neurological outcomes. Then we witnessed the full recovery of a cardiac arrest patient after implementing TTM. Now our perception of TTM is much improved" (M)

"The power of media is huge. It is important that the survivor stories are reported to the hospital marketing team to inform many people" (A)

Interdisciplinary and interprofessional collaboration

Departmental collaboration

Difficulty in interdisciplinary collaboration Conflict of department leadership

$\begin{array}{ll}\text { Interprofessional } & \text { Lack of } \\ \text { teamwork } & \text { interprofessional } \\ & \text { education }\end{array}$

Organisational resources and processes

Human

resources Lack of specialists or specialised teams High workloads

\section{Sharing experiences through conferences}

Collaborative and supportive hospital culture Multidisciplinary team approach

Specialists or specialised teams Consulting services

"Lack of manpower is the most important barrier in our hospital. Post-cardiac arrest care was one of most difficult jobs in the ED last year but the situation was slightly improved after the use of a new cooling machine this year. However, I still think that manpower is the most difficult problem" (A)

"Most of the interdepartmental conflicts resulted from a limited manpower issue. To overcome this problem, we overstepped our budgetary boundaries to purchase a cooling machine equipped with an auto-regulation system. By purchasing the cooling machine, a significant part of the manpower problem was resolved" $(M)$

"The most important facilitator is the cooling device. We cannot leave out equipment in this discussion. We can use the cooling equipment to save other manpower. It is also significant that the cooling equipment greatly decreased the time-consuming factor of conventionally using ice packs..." (A) "From a long-term perspective, I think that an appropriate medical treatment fee should be set for TTM after cardiac arrest. Although most of the relevant professionals currently work hard with passion, I think that a fee associated with the equipment and treatment must be established for other departments to readily accept the treatment" $(M)$ 
Table 3 Continued

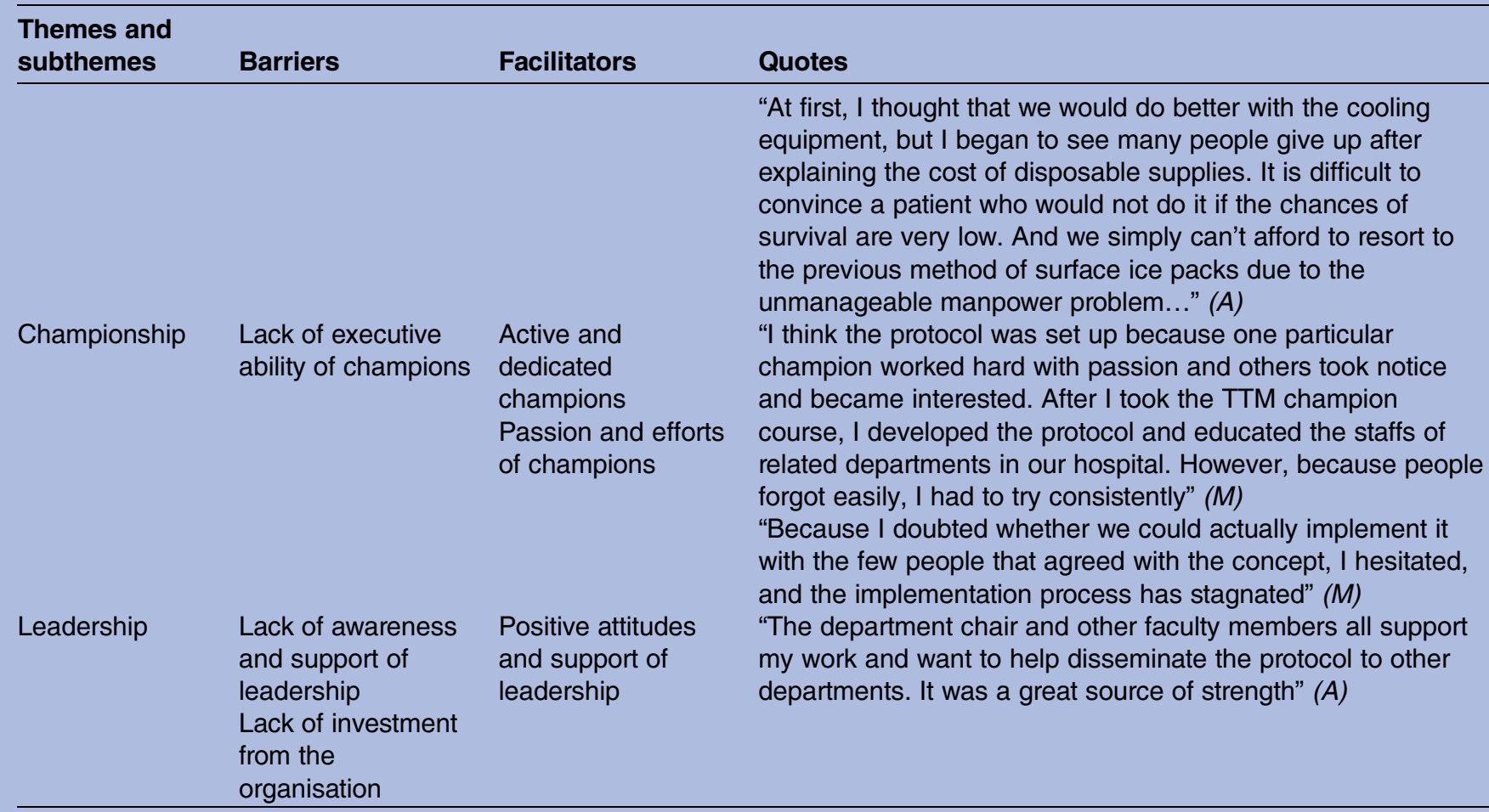

(A) indicates a participant who had the role of acting champion; $(M)$ indicates a participant who had the role of managerial champion; TTM, targeted temperature management.

protocol and believed that the perceptions of other physicians and nurses in the related departments are definitely changing. However, participants with limited TTM experience or those who experienced only cases with poor outcomes adapted the TTM but could not proceed to adhere to the protocol. Furthermore, the barriers related to organisational resources such as human resources and costs did not change dramatically even over time. However, some participants from hospitals that purchased cooling equipment with an automated feedback function during the study period stated that the equipment ameliorated manpower problems.

\section{DISCUSSION}

In this longitudinal qualitative study, we identified three themes related to barriers to and facilitators of implementing TTM guidelines for cardiac arrest survivors. The themes can also be explained by categorising them at the individual, team and organisational levels.

The first theme-healthcare professionals' perceptions of the guidelines and protocols-was categorised as a barrier and facilitator at the individual level. When this theme was examined with the awareness-toadherence model of the steps to clinical guideline compliance by Pathman et $a l^{21}$ and with the conceptual

Table 4 Top five barriers and facilitators perceived by managerial and acting champions

\begin{tabular}{|c|c|c|}
\hline Type of champion & Barriers & Facilitators \\
\hline $\begin{array}{l}\text { Managerial } \\
\text { champion }\end{array}$ & $\begin{array}{l}\text { 1. Difficulty in interdisciplinary collaboration } \\
\text { 2. Lack of equipment with an auto-feedback } \\
\text { function } \\
\text { 3. Lack of awareness and agreement with } \\
\text { guidelines } \\
\text { 4. Low medical treatment fees } \\
\text { 5. High cost of cooling equipment and supplies }\end{array}$ & $\begin{array}{l}\text { 1. Collaborative and supportive hospital culture } \\
\text { 2. Educational activities } \\
\text { 3. Publicity for successfully treated cases } \\
\text { 4. Passion and efforts of champions } \\
\text { 5. Cooling equipment with an auto-feedback function }\end{array}$ \\
\hline Acting champion & $\begin{array}{l}\text { 1. High workload demands } \\
\text { 2. Lack of equipment with an auto-feedback } \\
\text { function } \\
\text { 3. Difficulty in interdisciplinary collaboration } \\
\text { 4. Lack of interprofessional education } \\
\text { 5. High cost of cooling equipment and supplies }\end{array}$ & $\begin{array}{l}\text { 1. Cooling equipment with an auto-feedback function } \\
\text { 2. Publicity for successfully treated cases } \\
\text { 3. Educational activities } \\
\text { 4. Passion and efforts of champions } \\
\text { 5. Collaborative and supportive hospital culture }\end{array}$ \\
\hline
\end{tabular}


framework by Cabana et al, ${ }^{13}$ our study revealed that many clinicians were not familiar with the current guidelines (ie, in the awareness step) or had a negative attitude towards the guidelines (ie, in the agreement step) during the early implementation period. This lack of knowledge might have influenced the development of a negative attitude towards the guidelines, thus serving as a barrier to TTM implementation. Such barriers were also primarily identified by Toma et al, ${ }^{12}$ who studied clinical champions working in EDs and ICUs in Canada. Thus, local implementation efforts such as development and education of the protocol and order set by hospital champions are crucial for overcoming these barriers. In a recently randomised controlled trial, simple passive quality improvement interventions such as education, generic protocols, an order set and local champions significantly increased the rates of achieving successful TTM following OHCA in a large network of hospitals in Canada. $^{22}$

Changes in the recommendation level or grade in the new practice guidelines can lead to changes in the perceptions and attitudes of healthcare professionals. ${ }^{23}$ We also identified that healthcare professionals' internal barriers were influenced by guideline changes and improved with the accumulation of successful clinical experiences in our study. Therefore, during the early implementation period, it would be reasonable that the hospital team applied TTM preferentially to the most beneficial patients such as OHCA with shockable rhythms to accumulate their successful experiences. This preferential application may be an important step towards expanding TTM implementation.

The second theme-interdisciplinary and interprofessional collaboration-is a barrier and facilitator at the team level; it is especially important when practice guidelines for relatively complicated and time-sensitive acute diseases are used in a hospital with limited resources. ${ }^{24}{ }^{25}$ Currently, TTM after cardiac arrest has been implemented in EDs in many countries, including South Korea. ${ }^{26}$ Thus, the collaboration between physician champions and ED nurses is important for successful TTM implementation.

Furthermore, since postcardiac arrest care includes TTM, early coronary intervention, advanced critical care and neurological prognostication, close relationships among the related departments are critical. Similar barriers can probably be identified in many other implementations of new guidelines in the ICU, especially in patients who 'fall between' several specialties. ${ }^{27}$ Toma et $a l^{12}$ also identified this second theme as an integral aspect of the uptake process. They emphasised that interprofessional and interdisciplinary education will be required to coordinate implementation efforts and ensure a unified approach. Similarly, communication challenges across disciplines and lack of education were the two most emphasised barriers in a recent report from the USA. ${ }^{28}$ In our study, the second theme was a barrier that was most commonly mentioned by the managerial champions; they agreed that this barrier can be overcome through a supportive and cooperative culture in the hospital and by multidisciplinary conferences. Our findings are also consistent with those of Wang et $a l,{ }^{29}$ who emphasised that one of the key elements of implementing new TTM programmes is frequent multidisciplinary meetings involving all stakeholders.

The last theme-organisational resources and processes-is a barrier and facilitator at the system level that is commonly articulated when implementing complicated practice guidelines in the ED or ICU. ${ }^{24} 25$ In particular, this theme can be an important barrier in hospitals where manpower in the ED or ICU is relatively lacking. Although cooling equipment itself is a dependent factor associated with clinical outcomes, cooling equipment with an automated feedback function may facilitate TTM implementation in some hospitals.

Lack of personnel with expertise in postcardiac arrest care, one of the barriers to TTM implementation, may be resolved by creating an internal consultation service. Such solutions have been reported to be useful in implementing guidelines for severe sepsis and septic shock, as well as postcardiac arrest care. ${ }^{25}{ }^{30}$ In addition, championship and leadership within a hospital were also identified as important barriers and facilitators in our study. Wang et $a l^{29}$ emphasise that the entire hospital should collaboratively make an effort based on leadership and championship to implement postcardiac arrest TTM using a rapid-response-system approach.

\section{Study strengths and limitations}

A major strength of our study is that the facilitators to implementing the guidelines for TTM after cardiac arrest were also investigated. Notably, our study also adds longitudinal changes in the perceived barriers and facilitators over the early implementation period. Furthermore, we also identified the differences in the perceived facilitators and barriers between the champions who have different roles and responsibilities in the respective hospitals.

Nevertheless, this study has certain limitations. First, we recruited motivated hospital resuscitation champions who were the most likely to work in TTM implementation at each hospital purposively. Since most of the hospital resuscitation champions for postcardiac arrest care and TTM in South Korea are emergency physicians, only emergency physicians participated in this study. Thus, our findings may not reflect the perspectives of nursing champions or physician champions in other departments, especially intensive care physicians. Thus, further studies including multidisciplinary inputs are required. Second, the guidelines for TTM on which our study is based are over 5 years old. Although uncertainty exists regarding the indications, specific temperature target, TTM duration, and rewarming rate and 
method, currently selecting and maintaining a constant target temperature for the postcardiac arrest patients are still strongly recommended in new guidelines. Thus, the barriers and facilitators may also be helpful for clinical champions to implement new TTM guidelines. Further studies to determine the clinical benefit of reducing the barriers and applying the facilitators are also required. Third, since a longitudinal study is conducted to observe the selected participants over a certain period, the thematic saturation method was not an optimal sample size estimation method for the longitudinal qualitative study. Finally, our findings are from hospital resuscitation champions who worked in some hospitals with limited critical care human resources in an Asian country where TTM treatment fees are relatively low. Although most of the barriers identified in our study were similar to those of previous studies, clinicians in different countries or under different systems might have different perception and opinions.

\section{CONCLUSIONS}

In this longitudinal qualitative study, we identified barriers and facilitators perceived by hospital resuscitation champions in implementing TTM guidelines after cardiac arrest, which can be explained at the individual, team and organisational levels. Healthcare professionals' internal barriers to TTM implementation were influenced by guideline changes and were changed positively with the accumulation of successful clinical experiences during the early implementation period. Promoting interprofessional and interdisciplinary collaboration through educational activities and the use of cooling equipment with an automated feedback function may facilitate adherence to TTM guidelines, especially in hospitals with limited human resources in critical care.

Acknowledgements The authors thank Ji Sun Park for helping with the transcription and note-taking in focus group discussions. They also thank $\mathrm{Dr}$ Chan Woong Kim of Chung-Ang University Hospital for his kind advice on the qualitative analysis and critical review of our manuscript; and they thank American Journal Experts (AJE) for English language editing.

Contributors Y-MK and KNP conceived the study; Y-MK and SJJ designed the study. Y-MK and SJL conducted the data collection and managed the data, including the quality control. Y-MK, SJL and SJJ analysed the data; Y-MK and KNP interpreted the results. Y-MK drafted the manuscript, and all the authors contributed substantially to its revision.

Funding The authors wish to acknowledge the financial support of the Catholic Medical Center Research Foundation made in the program year of 2011.

Competing interests None declared.

Patient consent Obtained.

Ethics approval The study protocol was approved by the Seoul St. Mary's Hospital Institutional Research Ethics Board (KC11EIS0318).

Provenance and peer review Not commissioned; externally peer reviewed.

Data sharing statement No additional data are available.

Open Access This is an Open Access article distributed in accordance with the Creative Commons Attribution Non Commercial (CC BY-NC 4.0) license, which permits others to distribute, remix, adapt, build upon this work noncommercially, and license their derivative works on different terms, provided the original work is properly cited and the use is non-commercial. See: http:// creativecommons.org/licenses/by-nc/4.0/

\section{REFERENCES}

1. Peberdy MA, Callaway CW, Neumar RW, et al. Part 9: post-cardiac arrest care: 2010 American Heart Association Guidelines for Cardiopulmonary Resuscitation and Emergency Cardiovascular Care. Circulation 2010;122(Suppl 3):S768-86.

2. Deakin CD, Nolan JP, Soar J, et al. European Resuscitation Council Guidelines for Resuscitation 2010 Section 4. Adult advanced life support. Resuscitation 2010;81:1305-52.

3. Binks AC, Murphy RE, Prout RE, et al. Therapeutic hypothermia after cardiac arrest-implementation in UK intensive care units. Anaesthesia 2010;65:260-5.

4. Orban JC, Cattet F, Lefrant JY, et al. The practice of therapeutic hypothermia after cardiac arrest in France: a national survey. PLoS ONE 2012;7:e45284.

5. Vaahersalo J, Hiltunen P, Tiainen M, et al, FINNRESUSCI Study Group. Therapeutic hypothermia after out-of-hospital cardiac arrest in Finnish intensive care units: the FINNRESUSCI study. Intensive Care Med 2013;39:826-37.

6. Patel PV, John S, Garg RK, et al. Therapeutic hypothermia after cardiac arrest is underutilized in the United States. Ther Hypothermia Temp Manag 2011;1:199-203.

7. Krawczyk P, Kolodziej G, Szpyra B, et al. Implementation of therapeutic hypothermia after cardiac arrest in intensive care units in Poland. Kardiol Pol 2013;71:270-4.

8. Kim JY, Shin SD, Ro YS, et al. Post-resuscitation care and outcomes of out-of-hospital cardiac arrest: a nationwide propensity score-matching analysis. Resuscitation 2013;84:1068-77.

9. Gasparetto N, Scarpa D, Rossi S, et al. Therapeutic hypothermia in Italian intensive care units after 2010 resuscitation guidelines: still a lot to do. Resuscitation 2014;85:376-80.

10. Dainty KN, Brooks SC, Morrison LJ. Are the 2010 guidelines on cardiopulmonary resuscitation lost in translation? A call for increased focus on implementation science. Resuscitation 2013;84:422-5.

11. Brooks SC, Morrison LJ. Implementation of therapeutic hypothermia guidelines for post-cardiac arrest syndrome at a glacial pace: seeking guidance from the knowledge translation literature. Resuscitation 2008;77:286-92.

12. Toma A, Bensimon CM, Dainty $\mathrm{KN}$, et al. Perceived barriers to therapeutic hypothermia for patients resuscitated from cardiac arrest: a qualitative study of emergency department and critical care workers. Crit Care Med 2010;38:504-9.

13. Cabana MD, Rand CS, Powe NR, et al. Why don't physicians follow clinical practice guidelines? A framework for improvement. JAMA 1999;282:1458-65.

14. Morse JM. The significance of saturation. Qual Health Res 1995:5:147-9.

15. Soo S, Berta W, Baker GR. Role of champions in the implementation of patient safety practice change. Healthc Q 2009;12:123-8.

16. Silverman D. Doing qualitative research: a practical handbook. 2nd edn. Los Angeles, CA: Sage, 2005.

17. Graneheim UH, Lundman B. Qualitative content analysis in nursing research: concepts, procedures and measures to achieve trustworthiness. Nurse Educ Today 2004;24:105-12.

18. Braun V, Clarke V. Using thematic analysis in psychology. Qual Res Psychol 2006;3:77-101.

19. Stevens PE. Focus group: collecting aggregate-level data to understand community health phenomena. Public Health Nurs 1996;13:170-6.

20. Pope C, Mays N. Qualitative research in healthcare. 3rd edn. Malden, MA: Blackwell Publishing, 2006.

21. Pathman DE, Konrad TR, Freed GL, et al. The awareness-to-adherence model of the steps to clinical guideline compliance-the case of pediatric vaccine recommendations. Med Care 1996;34:873-89.

22. Morrison LJ, Brooks SC, Dainty KN, et al. Improving use of targeted temperature management after out-of-hospital cardiac arrest: a stepped wedge cluster randomized controlled trial. Crit Care Med 2015;43:954-64.

23. Leape LL, Weissman JS, Schneider EC, et al. Adherence to practice guidelines: the role of specialty society guidelines. Am Heart $J$ 2003;145:19-26.

24. Carlbom DJ, Rubenfeld GD. Barriers to implementing protocol-based sepsis resuscitation in the emergency departmentresults of a national survey. Crit Care Med 2007;35:2525-32. 
25. Mikkelsen ME, Gaieski DF, Goyal M, et al. Factors associated with nonadherence to early goal-directed therapy in the ED. Chest 2010;138:551-8.

26. Patil S, Bhayani S, Denton JM, et al. Therapeutic hypothermia for out-of-hospital cardiac arrest: implementation in a district general hospital emergency department. Emerg Med J 2011;28: 970-3.

27. Sinuff $T$, Cook D, Giacomini M, et al. Facilitating clinician adherence to guidelines in the intensive care unit: a multicenter, qualitative study. Crit Care Med 2007;35:2083-9.
28. Blewer AL, Delfin G, Leary M, et al. A structured educational intervention to improve targeted temperature management utilization following cardiac arrest. J Crit Care 2013;28:259-64.

29. Wang HE, Thomas JJ, James D, et al. Post-cardiac arrest therapeutic hypothermia: overcoming the barrier of workplace culture and other implementation lessons. Jt Comm J Qual Patient Saf 2011;37:425-32.

30. Rittenberger JC, Guyette FX, Tisherman SA, et al. Outcomes of a hospital-wide plan to improve care of comatose survivors of cardiac arrest. Resuscitation 2008;79:198-204. 